\title{
PERFORMANCE MANAGEMENT
}

\section{Wirkungen messen}

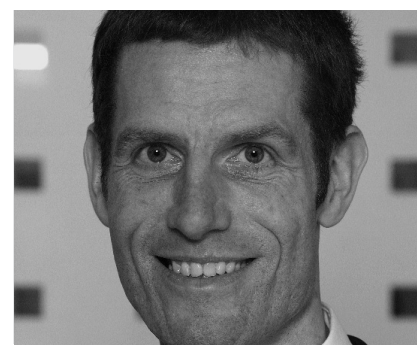

VON THOMAS EISENREICH Thomas Eisenreich ist Mitglied im Vorstand der Stiftung Alsterdorf in Hamburg, einem großen diakonischen Dienstleistungsunternehmen in Norddeutschland. Internet

http://www.alsterdorf.de

\author{
Neben den drei Qualitätsmerkmalen Struktur, Prozess \\ und Ergebnis gewinnt als vierte Dimension die der \\ "Wirkung" an Bedeutung. Das Konzept des Performan- \\ ce Managements will diese Entwicklung im Manage- \\ ment-Alltag berücksichtigen.
}

Die Suche der Non-Profit-Organisationen nach wirkungsorientierten Steuerungsinstrumenten zeigt sich in den zahlreichen Versuchen, solche zu beschreiben und in der Praxis umzusetzen. Neben den aktuellen Ansätzen des wirkungsorientierten Controllings, das von der International Group of Controlling (IGC) entwickelt wurde, und denen des Social Return on Invest (SROI) wird mit dem Performance Management ein weiteres Managementinstrument in die Branche gebracht. Was ist nun neu an dem Ansatz des Performance Managements gegenüber den beiden anderen genannten Instrumenten?

Der erste Teil des neuen Buches von Maria Laura Bono bietet das Rüstzeug für die weiteren drei Teile ihrer Ausführungen. Der Bogen ist weit gespannt. Von der Definition des Performance Managements bis hin zum Benchmarking reichen die gestreiften Themen. Wer sich das erste Mal dem Thema kennzahlenbasierter Steuerung nähert, erhält einen Überblick des notwendigen Know-how, um das Performance Management nutzen zu können. Ohne vertiefende Literatur zu den Themen Kennzahlen, Messung, Aufbau von Management-Informations-Systemen und Benchmarking wird es allerdings schwierig sein, sich fundiert in der Praxis auf den Weg zu machen.

Die Bausteine des Performance Managements werden im zweiten Teil intensiv erarbeitet. Zentrale Inhalte sind die Modellierung von Wirkungsketten und die Rollen der Stakeholder in NonProfit-Organisationen. Auch wenn in Teil 1 die unterschiedlichen Non-Profit-Organisationen klassifiziert werden, gilt es aufgrund der unterschiedlichen Auftragsfunktionen die Inhalte von Teil 2 auf die eigene Organisation zu übertragen. Der Transfer kann aufgrund der vielfältigen Auftragsziele auch nur ansatzweise in Buchform allumfassend erfolgen. Dies macht deutlich, dass zwischen den unterschiedlichen unternehmerischen oder nichtunternehmerischen Ausrichtungen der Non-Profit-Organisationen sehr differenzierte Managementbedarfe gibt.

Nach den ausführlichen Teilen eins und zwei folgt der eher kurz gehaltene Werkzeugkasten, in denen die Autorin den Transfer in die praktische Anwendung vollzieht. Hinsichtlich des Buchtitels geht aus den ersten beiden Teilen einiges verloren. So reduziert sich der Teil auf die Wirkungsketten und Kennzahlen. Letztere werden in einem kurzen Kennzahlenkatalog nebst den dazugehörigen Berechnungsformeln gelistet. In den Wirkungsketten finden sich die finanzwirtschaftlichen Kennzahlen des Katalogs jedoch nicht wieder. Damit bleibt leider die spannende Frage unbeantwortet, wie die Wirkung der Leistungen von Non-Profit-Organisationen mit den betriebswirtschaftlichen Steuerungsparametern korrespondieren. Dies wird auch im vierten Teil mit den Fallbeispielen nicht aufgelöst.

Entsprechend der von Maria Laura Bono gewählten Definition des Perfor- 
mance Managements als »das Erfüllungsniveau ... welches durch ein Individuum, ein Team, eine Organisation oder einen Prozess erreicht wird « konzentriert sie sich auf ein mehrdimensionales

Qualitätsmanagement. Führungskräfte in Non-Profit-Organisationen, die derzeit die harten wirtschaftlichen Einschnitte seitens der Kostenträger erleben und damit den zunehmenden Spagat zwischen Mittelverknappung und Qualitätserhaltung lösen müssen, werden die wirtschaftliche (harte) Dimension vermissen. Dies gilt insbesondere für die Organisationen, deren Leistungen pflegesatzrefinanziert sind und damit einen erheblichen Steuerungsbedarf hinsichtlich der Prozesskosten und Prozesserlösen haben.

Das Buch zum Performance Management empfiehlt sich für die Organisationseinheiten einer Non Profit-Organisation, die sich auf dem Weg machen möchten, das eigene Qualitätsmanagement neben den bekannten drei Qualitätsdimensionen - Struktur, Prozess, Ergebnis - um die spannende vierte Dimension der Wirkung zu bereichern. Spannend ist es sicherlich, die Ansätze des wirkungsorientierten Controllings und des Social Return on Investment mit dem Performance Management zu koppeln. Damit würden die betriebswirtschaftlichen Steuerungsdimensionen ebenfalls um die Wirkung der eingesetzten harten Finanzmittel auf die Performance für die Stakeholder und die Gesellschaft ergänzt. Zudem kann das Performance Management mit den Wirkungsketten der fehlende Baustein sein, die Messung des Social Return on Investment aus der wissenschaftlichen Dimension in den strategischen Werkzeugkasten der sozialen Organisationen zu holen.

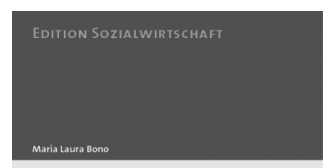

Performance

Management in NPOs

steuerung im Dienste sozialer Ziele

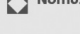

ISBN 978-3-8329-5082-8.

\section{Migranten und ihre Familien im Hartz-IV-Bezug}

\section{- ein für die Integrationspolitik ungenutztes Feld}

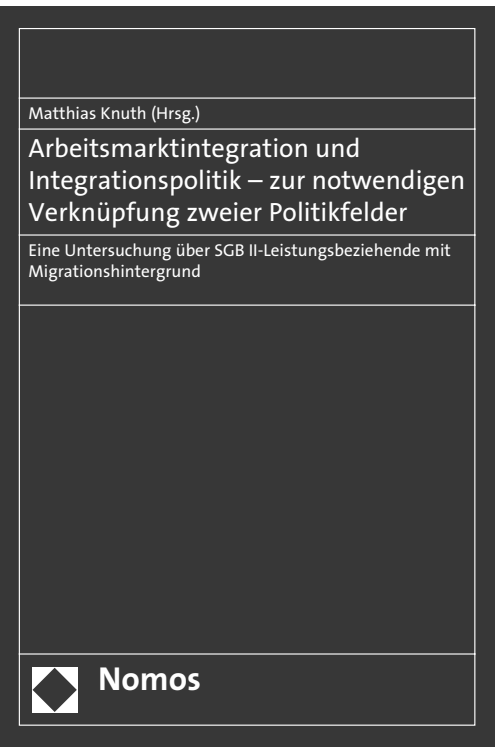

Migranten bilden unter den Hartz-IV-Empfängern die größte Gruppe. Sie wurden jedoch bei der Konzipierung der Hartz-Reformen völlig vergessen. Der Sammelband beruht auf einer vom Bundesministerium für Arbeit und Soziales in Auftrag gegebenen Untersuchung. Auf der Grundlage einer repräsentativen Befragung von Hartz-IV-Empfängern, durch Auswertung von Geschäftsdaten der Bundesagentur für Arbeit und aufgrund von Fallstudien vermitteln die an der Untersuchung beteiligten Forscherinnen und Forscher ein facettenreiches Bild von Lebenslage und Potenzialen von Zuwanderern im Hartz-IV-Bezug, vom Umgang der Jobcenter mit ihnen, von der Realität des „Förderns und Forderns“ und von seinen begrenzten Wirkungen.

Wenig geeignet zur Skandalisierung, geben die Befunde viel Stoff zum Nachdenken - z.B. über den Zusammenhang zwischen Personalpolitik in öffentlichen Verwaltungen und den Versäumnissen der Integrationspolitik, oder über den Widerspruch zwischen der Förderung des männlichen Haupternährermodells im Steuer- und Sozialversicherungssystem für den wirtschaftlich stärkeren Teil der Bevölkerung und der Forderung nach uneingeschränkter Erwerbsbereitschaft beider Partner im Falle von Bedürftigkeit.

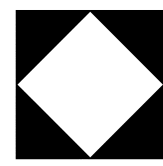

\section{Nomos}

Bitte bestellen Sie im Buchhandel oder versandkostenfrei unter $\longrightarrow$ www.nomos-shop.de 